\title{
Osimertinib in Pretreated T790M-Positive Advanced Non-Small-Cell Lung Cancer: AURA Study Phase II Extension Component
}

James Chih-Hsin Yang, Myung-Ju Ahn, Dong-Wan Kim, Suresh S. Ramalingam, Lecia V. Sequist, Wu-Chou Su, Sang-We Kim, Joo-Hang Kim, David Planchard, Enriqueta Felip, Fiona Blackhall, Daniel Haggstrom, Kiyotaka Yoh, Silvia Novello, Kathryn Gold, Tomonori Hirashima, Chia-Chi Lin, Helen Mann, Mireille Cantarini, Serban Ghiorghiu, and Pasi A. Jänne

Author affiliations and support information (if applicable) appear at the end of this article.

Published at jco.org on February 21, 2017. Clinical trial information: NCT01802632.

Corresponding author: James Chih-Hsin Yang, MD, PhD, National Taiwan University Hospital, National Taiwan University Cancer Center, 7 Chung-Shan South Rd, Taipei, 100 Taiwan, Republic of China; e-mail: chihyang@ntu.edu.tw.

(c) 2017 by American Society of Clinical Oncology

0732-183X/17/3599-1/\$20.00
ASSOCIATED CONTENT

Q Appendix DOI: $10.1200 / J C 0.2016 .70 .3223$

Data Supplement DOI: $10.1200 / J C 0.2016 .70 .3223$ DOI: $10.1200 / J C O .2016 .70 .3223$

\section{$\begin{array}{lllllllll}\text { A } & \text { B } & \mathbf{S} & \mathbf{T} & \mathbf{R} & \mathbf{A} & \mathbf{C} & \mathbf{T}\end{array}$}

\section{Purpose}

Osimertinib is an irreversible epidermal growth factor receptor (EGFR) tyrosine kinase inhibitor (TKI) selective for both EGFR-TKI sensitizing (EGFRm) and T790M resistance mutations. AURA (NCT01802632) is a phase I/II clinical trial to determine the dose, safety, and efficacy of osimertinib. This article reports the results from the phase II extension component.

\section{Patients and Methods}

Patients with EGFR-TKI-pretreated EGFRm- and T790M-positive advanced non-small-cell lung cancer (NSCLC) received once-daily osimertinib $80 \mathrm{mg}$. T790M status was confirmed by central testing from a tumor sample taken after the most recent disease progression. Patients with asymptomatic, stable CNS metastases that did not require corticosteroids were allowed to enroll. The primary end point was objective response rate (ORR) by independent radiology assessment. Secondary end points were disease control rate, duration of response, progression-free survival (PFS), and safety. Patient-reported outcomes comprised an exploratory objective.

\section{Results}

In total, 201 patients received treatment, with a median treatment duration of 13.2 months at the time of data cutoff (November 1, 2015). In evaluable patients ( $n=198)$, ORR was $62 \%(95 \% \mathrm{Cl}, 54 \%$ to $68 \%)$, and the disease control rate was $90 \%(95 \% \mathrm{Cl}, 85$ to 94$)$. Median duration of response in 122 responding patients was 15.2 months $(95 \% \mathrm{Cl}, 11.3$ to not calculable). Median PFS was 12.3 months ( $95 \% \mathrm{Cl}, 9.5$ to 13.8). The most common possibly causally related adverse events (investigator assessed) were diarrhea (43\%; grade $\geq 3,<1 \%$ ) and rash (grouped terms; $40 \%$; grade $\geq 3,<1 \%)$. Interstitial lung disease (grouped terms) was reported in eight patients (4\%; grade $1, \mathrm{n}=2$; grade $3, \mathrm{n}=3$; grade $5, \mathrm{n}=3$ ).

\section{Conclusion}

In patients with EGFRm T790M advanced NSCLC who progress after EGFR-TKI treatment, osimertinib provides a high ORR, encouraging PFS, and durable response.

\section{J Clin Oncol 35. (C) 2017 by American Society of Clinical Oncology}

\section{INTRODUCTION}

Epidermal growth factor receptor (EGFR) tyrosine kinase inhibitors (TKIs) are the recommended first-line treatment of patients with advanced lung cancer that harbors an activating EGFR mutation (EGFRm), ${ }^{1,2}$ which is supported by results from several phase III studies. ${ }^{3-8} \mathrm{De}-$ spite high initial responses to first-line EGFRTKIs and a median progression-free survival (PFS) of 10 to 14 months, resistance eventually develops in most, if not all, patients. The most common mechanism of acquired resistance to EGFR-TKIs, the EGFR p.Thr790Met point mutation (T790M), can be detected in $50 \%$ to $60 \%$ of tissue biopsy samples taken after acquired resistance. $^{9-12}$

Osimertinib is an oral, irreversible EGFRTKI selective for both EGFRm and T790M resistance mutations. ${ }^{13}$ The phase I component of the osimertinib AURA (NCT01802632) study showed clinical activity across oral doses of 20 to $240 \mathrm{mg} /$ day. $^{14}$ The optimal dose chosen for 
further phase II evaluation was $80 \mathrm{mg}$ once daily. The phase I study results suggested that both patients with T790M-positive nonsmall-cell lung cancer (NSCLC) and patients with T790M not detected derive benefit from osimertinib treatment but with a much higher response rate in patients with T790M-positive disease (objective response rate [ORR], 61\% $v 21 \%$ ). AURA extension is the phase II component of the AURA study. We report the treatment outcomes, including ORR, by blinded independent central review (BICR), duration of response (DoR), and PFS of patients in the AURA extension phase II study.

\section{PATIENTS AND METHODS}

\section{Study Design and Participants}

The AURA study was a phase I/II, open-label, multicenter study of osimertinib in patients with advanced NSCLC who had progressed after therapy with an EGFR-TKI agent (with or without additional anticancer regimens). Patients were at least 18 years of age ( $\geq 20$ years in Japan) with a histologically or cytologically confirmed diagnosis of NSCLC that harbored confirmed EGFR-TKI sensitizing EGFRm and were enrolled across 46 study centers in Japan (16), the United States (seven), South Korea (four), Australia (three), France (three), Germany (three), Spain (three), Italy (three), Taiwan (two), and the United Kingdom (two).

The AURA study consisted of dose escalation and dose expansion in phase I with the capsule formulation of osimertinib (methods and preliminary results have been previously reported $)^{14}$ and a phase II extension component with the tablet formulation at the recommended phase II 80-mg once-daily dose. Two cohorts comprised the phase II extension component: patients whose disease had progressed (confirmed by radiologic documentation) either after only one prior therapy with an EGFRTKI (second line) or after treatment with at least two lines of prior therapy, including at least one EGFR-TKI (third line or more).

Eligibility criteria are provided in detail in the Appendix (online only). Briefly, patients were required to have a WHO performance status of 0 to 1 and acceptable organ function. Patients with CNS metastases could be enrolled if the disease was asymptomatic, stable, and not requiring corticosteroids for at least 4 weeks before the first dose. Prospective confirmation of T790M mutation status was by a central laboratory that used the cobas EGFR Mutation Test (Roche Molecular Systems, Pleasanton, CA; Appendix) from a tissue biopsy specimen taken after disease progression on the most recent treatment regimen (irrespective of whether EGFR-TKI or chemotherapy).

All participating sites required approval from the independent institutional review board/independent ethics committee. The study was performed in accordance with the ethical principles that have their origin in the Declaration of Helsinki and that are consistent with International Conference on Harmonization/Good Clinical Practice and applicable regulatory requirements. All patients provided written informed consent before their participation in the study. The protocol was designed by the sponsor (AstraZeneca) and the study investigators.

\section{Procedure}

Eligible patients received $80 \mathrm{mg}$ osimertinib orally once daily and continued treatment until Response Evaluation Criteria in Solid Tumors version 1.1 (RECIST1.1)-defined progression or until a treatment discontinuation criterion was met. Patients could continue to receive osimertinib beyond RECIST1.1-defined progression if they continued to show clinical benefit as judged by the investigator. If osimertinib was discontinued for reasons other than disease progression, the patient continued response assessment every 6 weeks until disease progression.

The primary end point of the AURA phase II extension study was ORR by BICR. Secondary end points were DoR, disease control rate (DCR), tumor shrinkage, PFS, overall survival (OS), safety, tolerability, and pharmacokinetics. Collection of patient-reported outcomes (PROs) and quality of life were exploratory objectives. DoR, DCR, tumor shrinkage, and PFS were determined by using RECIST1.1 assessed by BICR. Adverse events (AEs) were graded according to the National Cancer Institute Common Terminology Criteria for Adverse Events (version 4). The Appendix provides further details on procedures related to AEs and the collection of PROs.

\section{Statistical Analysis}

The full analysis set was defined as all patients enrolled who received at least one dose of study treatment. The evaluable for response analysis set was defined as all patients who received at least one dose of study treatment and had measurable disease at baseline according to BICR. CNS efficacy was assessed in an evaluable for CNS response analysis set, which included patients with at least one measurable CNS lesion on baseline brain scan (RECIST1.1) by BICR (assessed by neuroradiologists). The primary efficacy end point variable ORR by BICR (according to RECIST1.1) was defined as the percentage of patients with at least one visit response of complete response or partial response confirmed at least 4 weeks later. (Definitions of PFS and DoR are provided in the Appendix.) No formal statistical analysis was done. The study was sized to recruit approximately 175 patients to estimate an ORR with $95 \%$ CI within $\pm 8 \%$ and to adequately assess safety and tolerability.

Statistical analyses were performed by Pharmaceutical Product Development (Wilmington, NC). All calculations were performed with SAS 9.2 software (SAS Institute, Cary, NC) unless otherwise stated. We report a November 1, 2015, data cutoff.

\section{RESULTS}

\section{Demographics}

Between May 14, 2014, and November 1, 2015, 401 patients were screened, and 327 patients' samples were eligible for T790M testing, of which 324 (99\%) gave a valid test result; 207 (64\%) had positive test results for T790M. The T790M detection rate did not vary by last prior EGFR-TKI treatment (gefitinib, 69\%; erlotinib, 68\%; afatinib, 68\%; Appendix Table A1, online only).

Two hundred one patients received osimertinib treatment (61 as second-line therapy and 140 as third-line or more therapy; Fig 1); three patients had no measurable disease at baseline by BICR and were excluded from the evaluable for response set $(n=198)$. Baseline characteristics are listed in Table 1. Twenty-five patients were included in a CNS response analysis set.

\section{Duration of Treatment}

At the time of analysis, the median duration of exposure was 13.2 months (range, 0.1 to 17.6 months). By investigator assessment, 106 patients were known to be alive after radiologic progression of whom 79 continued to receive osimertinib for at least 7 days after their first radiologic progression for a median duration of 3.4 months (Fig 1). Forty-eight of the 106 patients received at least one subsequent cancer therapy after progression (Fig 1).

\section{Efficacy}

Tumor response. One hundred ninety-eight patients were evaluable for response by BICR. ORR was 62\% (122 of 198 patients; $95 \%$ CI, 54 to 68), and DCR was $90 \%$ (179 of 198 patients; $95 \%$ CI, 85 to 94 ). ORRs ranging from $53 \%$ to $68 \%$ were observed across all presented predefined subgroups (Fig 2). ORRs were similar between the second-line and third-line or more cohorts 


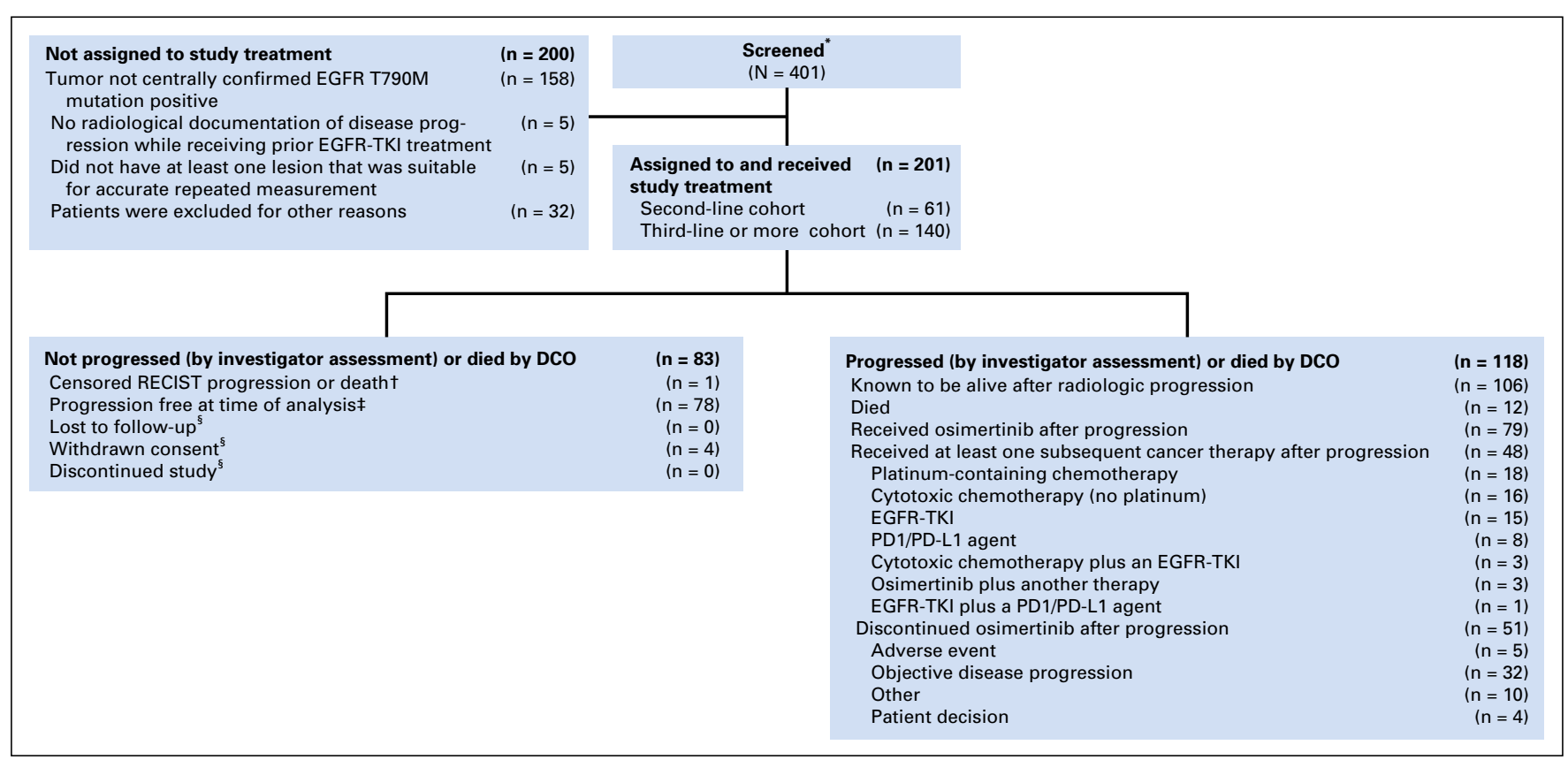

Fig 1. Patient disposition. All treatment decisions were based on investigator interpretation of patient disease status. ${ }^{*}$ Informed consent received. Patients could have

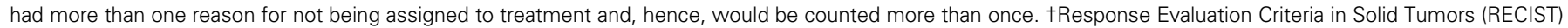

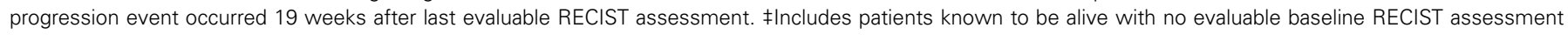
(censored at day 0). §Patients at last evaluable RECIST assessment. DCO, data cutoff; EGFR, epidermal growth factor receptor; TKI, tyrosine kinase inhibitor.

(62\% and 61\%) and by common EGFR-TKI sensitizing mutation status (exon 19 deletion, 64\%; L858R, 57\%), respectively (Fig 2). Post hoc analyses of ORR by last prior EGFR-TKI treatment were as follows: gefitinib, 68\% (64 of 94 patients; 95\% CI, 58 to 77); erlotinib, 57\% (56 of 99 patients; $95 \%$ CI, 46 to 67 ); and afatinib/ dacomitinib, $50 \%$ (two of four patients; $95 \%$ CI, 7 to 93 ).

Overall, $99(81 \%)$ of 122 responders had a documented initial response at their first scheduled follow-up RECIST1.1 scan at $6 \pm$ 1 weeks. Tumor shrinkage was seen in the majority of patients (94\%; Fig 3A). The mean best percentage change in target lesion size from baseline was $-42.7 \%$.

Of the 122 patients deemed to have an objective response, 46 (38\%) had subsequently progressed or died by the time of analysis. The median DoR was 15.2 months (95\% CI, 11.3 to not calculable [NC]; range, 1.4 to 15.3 months). The proportion of patients with an objective response who subsequently progressed or died was similar by last EGFR-TKI treatment received before osimertinib (gefitinib, 22 [34\%] of 64; erlotinib, 23 [41\%] of 56; afatinib/ dacomitinib, one [50\%] of two).

PFS and OS. At the time of data cutoff, 107 (53\%) of 201 patients had progressed or died (by BICR). One hundred four of these patients were on treatment at the time of progression; three had discontinued osimertinib before progression and, therefore, were not censored. Median PFS (by BICR) was 12.3 months (95\% CI, 9.5 to $13.8)$; 11.0 months ( $95 \% \mathrm{CI}, 6.7$ to $\mathrm{NC}$ ), and 12.4 months (95\% CI, 9.5 to 15.5$)$ in the second-line and third-line or more cohorts, respectively (Fig 4A). The proportion of patients progression free at 6,9 , and 12 months was $70 \%, 58 \%$, and $52 \%$, respectively.

Subset analysis of PFS by EGFRm status before the start of the study showed a nonsignificant trend toward longer PFS in patients who harbored an exon 19 deletion (median, 12.5 months; 95\% CI, 9.7 to NC) compared with L858R (median, 9.6 months; $95 \% \mathrm{CI}$,
6.9 to 13.8 ; Fig 4B). Similarly, a nonsignificant trend toward longer PFS was observed in Asian versus non-Asian patients (median, 12.6 months [95\% CI, 9.7 to 16.6] $v 9.7$ months [95\% CI, 7.0 to 13.8], respectively; Fig 4C). Median PFS by last prior EGFR-TKI treatment was 12.6 months (95\% CI, 9.5 to NC) for gefitinib and 11.0 months (95\% CI, 6.9 to 13.8) for erlotinib (Fig 4D).

Median PFS generally was consistent across other subgroups analyzed: age at screening ( $<65 v \geq 65$ years), smoking status (never $v$ ever), last treatment before osimertinib (EGFR-TKI $v$ non-EGFR-TKI), immediate prior EGFR-TKI treatment ( $<30$ days before osimertinib $v \geq 30$ days before osimertinib), and duration of most recent EGFR-TKI treatment ( $<6 v \geq 6$ months; Appendix Table A2, online only).

At the time of data cutoff, 54 patients (27\%) had died; hence, the median OS was not reached ( $95 \% \mathrm{CI}, 16.4$ to NC). The median follow-up for OS was 13.8 months. One-year survival rate was $79 \%$ (95\% CI, 72 to 84; Appendix Fig A1, online only).

Symptom resolution. Data from European Organization for Research and Treatment of Cancer questionnaires QLQ-LC13 and QLQ-C30 showed that patients had consistent and sustained improvements in key lung cancer symptoms, including dyspnea, cough, chest pain, and pain in arm or shoulder (Appendix Fig A2, online only). Sustained improvements in global health status and physical functioning domains also were recorded.

Osimertinib activity in patients with CNS metastases. Seventyfour patients had CNS metastases at entry. Median PFS was shorter in patients with CNS metastases than in those without (median, 7.1 months [ $95 \%$ CI, 4.2 to 12.3 ] $v 13.7$ months [ $95 \% \mathrm{CI}, 11.0$ to $\mathrm{NC}$ ], respectively; Appendix Fig A3, online only). In the CNS response analysis set, the ORR was $64 \%$ ( 16 of 25 patients; $95 \%$ CI, 43 to 82); four patients experienced a complete response and 12 patients a partial response. Tumor shrinkage was seen in the majority of patients (Fig 3B) 


\begin{tabular}{|c|c|c|c|}
\hline Characteristic & $\begin{array}{l}\text { Second Line, } \\
\text { No. (\%) }\end{array}$ & Third Line or More, No. (\%) & $\begin{array}{l}\text { Total, } \\
\text { No. (\%) }\end{array}$ \\
\hline No. of patients & 61 & 140 & 201 \\
\hline \multicolumn{4}{|l|}{ Age, years } \\
\hline Mean (SD) & $62.6(10)$ & $60.9(11)$ & $61.4(11)$ \\
\hline Median (range) & $61(45-89)$ & $63(37-84)$ & $62(37-89)$ \\
\hline \multicolumn{4}{|l|}{ Sex } \\
\hline Male & $20(33)$ & $48(34)$ & $68(34)$ \\
\hline Female & $41(67)$ & $92(66)$ & $133(66)$ \\
\hline \multicolumn{4}{|l|}{ Ethnicity } \\
\hline White & $24(41)$ & $52(37)$ & $76(38)$ \\
\hline Asian & $32(54)$ & $82(59)$ & $114(57)$ \\
\hline Black or African American & 0 & $1(1)$ & $1(<1)$ \\
\hline Other & $1(2)$ & $3(2)$ & $4(2)$ \\
\hline Not reported* & 2 (3) & $2(1)$ & $4(2)$ \\
\hline \multicolumn{4}{|l|}{ Smoking status } \\
\hline Never & $41(67)$ & $93(66)$ & $134(67)$ \\
\hline Former & $20(33)$ & $42(30)$ & $62(31)$ \\
\hline Current & 0 & $5(4)$ & $5(2)$ \\
\hline \multicolumn{4}{|l|}{ WHO performance status } \\
\hline 0 & $25(41)$ & $43(31)$ & 68 (34) \\
\hline 1 & $36(59)$ & $96(69)$ & $132(66)$ \\
\hline 2 & 0 & $1(1)$ & $1(<1) \dagger$ \\
\hline \multicolumn{4}{|l|}{ Histology } \\
\hline Adenocarcinoma & $60(98)$ & $135(96)$ & 195 (97) \\
\hline Adenosquamous carcinoma & 0 & $1(1)$ & $1(<1)$ \\
\hline Other & $1(2)$ & 4 (3) & $5(2)$ \\
\hline EGFR T790M mutation by cobas central test & 59 (97) & 138 (99) & $197(98) \ddagger$ \\
\hline \multicolumn{4}{|l|}{$\begin{array}{l}\text { EGFR mutations co-occurring with } \\
\text { T790M by cobas central test }\end{array}$} \\
\hline Exon 19 deletion & $44(72)$ & $98(70)$ & $142(71)$ \\
\hline L858R & $16(26)$ & $35(25)$ & $51(25)$ \\
\hline G719X & $1(2)$ & $3(2)$ & $4(2)$ \\
\hline S768I & 0 & $3(2)$ & $3(1)$ \\
\hline Exon 20 insertion & $1(2)$ & $1(1)$ & $2(1)$ \\
\hline T790M only & 0 & $5(4)$ & $5(2)$ \\
\hline \multicolumn{4}{|l|}{ Overall disease classification } \\
\hline Metastatic & $59(97)$ & 138 (99) & $197(98)$ \\
\hline Locally advanced & $2(3)$ & $2(1)$ & $4(2)$ \\
\hline \multicolumn{4}{|l|}{ Metastases } \\
\hline CNS§ & $14(23)$ & $60(43)$ & 74 (37) \\
\hline Visceral & $50(82)$ & $123(88)$ & $173(86)$ \\
\hline Median No. of prior regimens (range) & 1 & $3(2-11)$ & $2(1-11)$ \\
\hline \multicolumn{4}{|l|}{ Prior treatment } \\
\hline \multicolumn{4}{|l|}{ First-generation EGFR-TKIs } \\
\hline Gefitinib & $32(52)$ & $85(61)$ & $117(58)$ \\
\hline Erlotinib & $28(46)$ & $88(63)$ & $116(58)$ \\
\hline \multicolumn{4}{|l|}{ Second-generation EGFR-TKIs } \\
\hline Afatinib & $0(0)$ & $36(26)$ & $36(18)$ \\
\hline Afatinib + cetuximab & & 4 (3) & $4(2)$ \\
\hline Dacomitinib & $1(2)$ & $3(2)$ & $4(2)$ \\
\hline Other EGFR-TKI & 0 & $5(4)$ & $5(2)$ \\
\hline Platinum-containing doublet chemotherapy & 0 & $122(87)$ & $122(61)$ \\
\hline Platinum-containing doublet chemotherapy + bevacizumab & 0 & $25(18)$ & 25 (12) \\
\hline \multicolumn{4}{|c|}{$\begin{array}{l}\text { Abbreviations: EGFR, epidermal growth factor receptor; TKI, tyrosine kinase inhibitor. } \\
\text { * Some countries do not allow collection of ethnicity data. } \\
\text { tProtocol deviation: inclusion criteria state that patients should have a WHO performance status of } 0 \text { or } 1 . \\
\text { fThree patients with EGFRT790M mutation status not detected (negative) and one patient not centrally tested entered the study; consequently, these were considerec } \\
\text { important protocol deviations. Of the three patients with no T790M detected by central testing, one had detectable exon } 19 \text { deletion, one had detectable L858R, and one } \\
\text { had detectable exon } 19 \text { deletion/S768I. } \\
\text { \$CNS metastases were determined programmatically from baseline data: patients with a CNS metastatic site, those who reported prior radiotherapy in anatomic } \\
\text { locations unequivocally in the CNS, and/or those who reported surgical excision of tumor from anatomic locations unequivocally in the CNS. }\end{array}$} \\
\hline
\end{tabular}

\section{Safety}

Nearly all patients reported at least one AE (199 [99\%] of 201), with 185 (92\%) reporting at least one AE possibly casually related to osimertinib treatment. A summary of safety and all-cause
AEs are listed in Appendix Tables A3 and A4 (online only). The most common possibly causally related AEs (by investigator assessment) were diarrhea, rash (grouped terms), paronychia (grouped terms), and dry skin (grouped terms; Table 2). The 


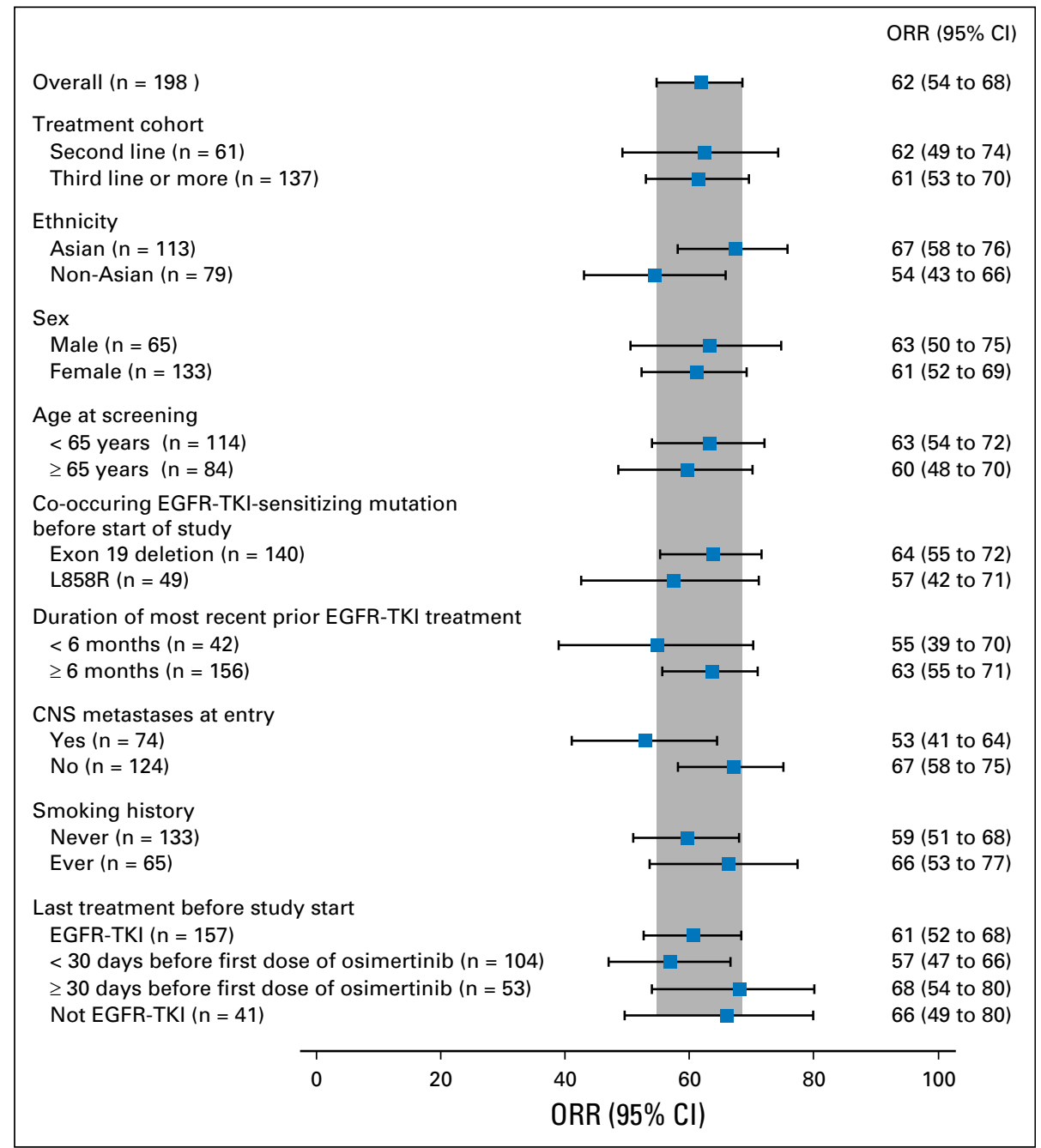

Fig 2. Objective response rate (ORR) by central review by subgroup in the evaluable for response set. The $95 \% \mathrm{Cls}$ were calculated by using ClopperPearson exact method for binomial proportions. The gray band represents the $95 \% \mathrm{Cl}$ for the overall patient ORR. EGFR, epidermal growth factor receptor; TKI, tyrosine kinase inhibitor. majority of these possibly causally related AEs were mild (grade 1 in severity).

Interstitial lung disease (ILD) grouped term AEs were reported in eight patients, all deemed possibly causally related (4\%; grade $1, \mathrm{n}=2$; grade $3, \mathrm{n}=3$; grade $5, \mathrm{n}=3$ ). Three AEs resolved, two were ongoing at the time of data cutoff, and three were reported as fatal (two reports of ILD and one of pneumonitis). Three $(4 \%)$ of 76 AEs were reported in white patients, three $(9 \%)$ of 35 in Japanese patients, and two (4\%) of 45 in non-Japanese Asian patients. The median time to onset for ILD grouped term AEs was 5.1 months. QT prolongation AEs were recorded in six patients ( $3 \%$; grade $1, \mathrm{n}=5$; grade $2, \mathrm{n}=1$ ), possibly causally related in three patients $(2 \%$; grade $1, n=2$; grade $2, n=1)$. In two reports, QT prolongation led to a dose reduction.

Forty-three patients $(21 \%)$ experienced AEs that led to dose interruption of osimertinib treatment, and 10 patients (5\%) experienced AEs that led to dose reduction. Nine patients (3\%) discontinued osimertinib treatment as a result of possibly causally related AEs (seven because of ILD grouped terms [discontinuation mandated by protocol]; one because of neutrophil count decrease; and one because of decreased appetite, asthenia, diarrhea, and vomiting).

\section{DISCUSSION}

In this study, oral osimertinib $80 \mathrm{mg}$ once daily resulted in a high ORR and DCR with significant DoR and PFS in patients with EGFR-TKI-resistant EGFRm lung cancer with tumors harboring T790M. This finding is consistent with the previous analysis of the AURA phase I study that demonstrated high response rates across the 20- to 240-mg dose range of osimertinib in patients with T790M-positive tumors. ${ }^{14}$ The current study demonstrates a median PFS of 12.3 months and a DoR of 15.2 months in responding patients. The phase II study AURA2 (NCT02094261 [independent phase II study with a similar design conducted in different centers]) demonstrated a similar ORR and PFS, which further confirms the efficacy of osimertinib in patients who 


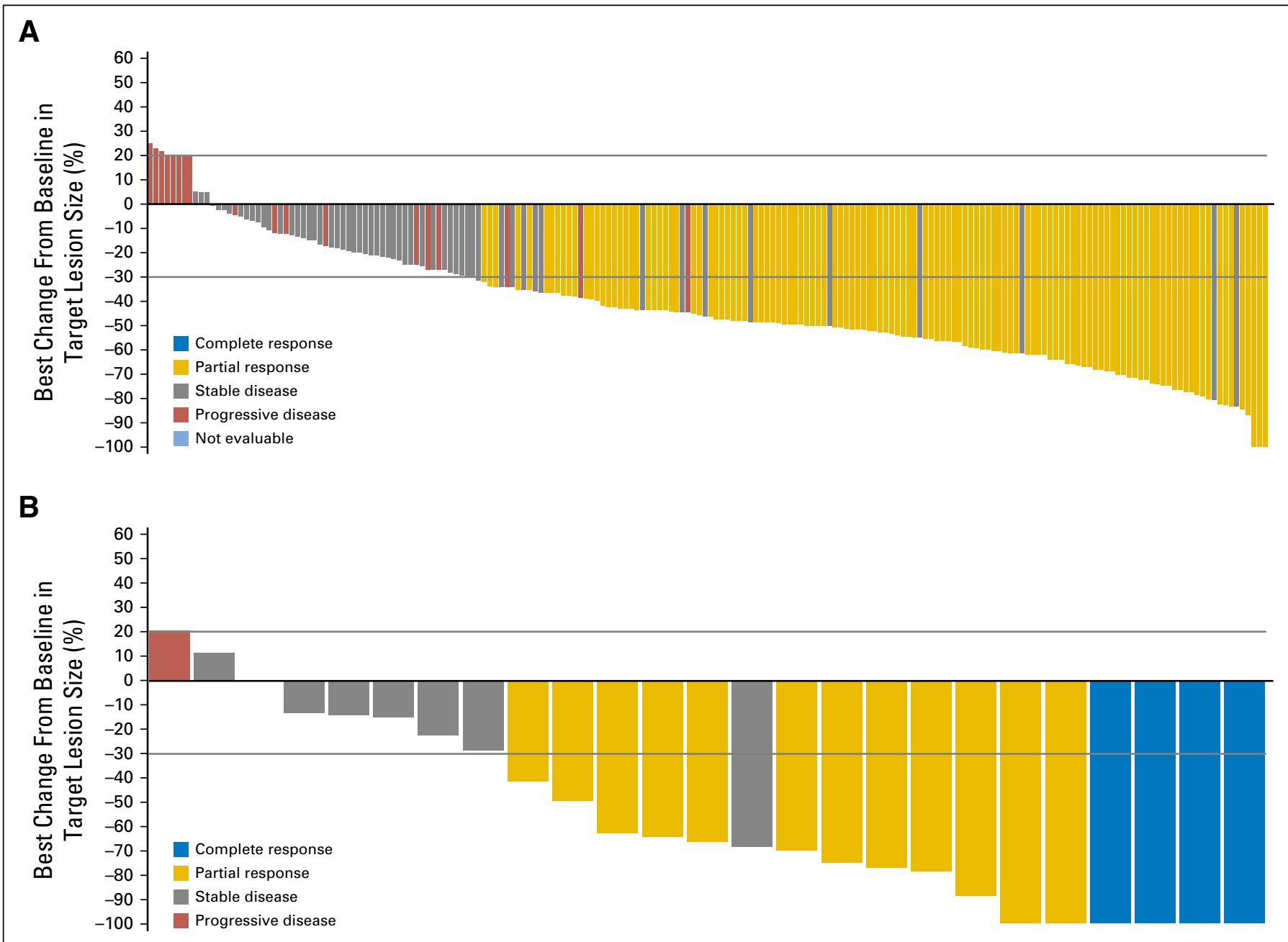

Fig 3. Best percentage change from baseline in target lesion size by central review in (A) the evaluable for response set and in (B) the CNS response analysis set. The line at $20 \%$ represents the boundary for determination of progressive disease, and the line at $-30 \%$ represents the boundary for determination of partial response.

harbor T790M. ${ }^{15}$ We also report encouraging systemic PFS with osimertinib in patients with CNS metastases and a high CNS response $(64 \%)$ in those with measurable CNS lesions. The latter is particularly relevant because new pharmacologic strategies are needed to treat brain metastases given the long-term complications of brain radiation. ${ }^{16}$

The standard recommendation for patients with EGFRm who progress after first-line EGFR-TKI treatment is chemotherapy, for which the maximum PFS reported to date is $<6$ months. ${ }^{17}$ For these patients, alternative treatment strategies have been explored. A study of treatment with paclitaxel and afatinib resulted in an ORR of $32 \%$ and PFS of 5.6 months. ${ }^{18}$ In a phase II study that evaluated afatinib in combination with cetuximab, the response rate in patients with T790M-positive tumors was $32 \%$ and the median PFS 4.7 months. ${ }^{19}$ However, a high rate of grade $3(44 \%)$ and $4(2 \%)$ AEs were reported with this combination. The ORR, PFS, and safety profile with osimertinib in both the AURA extension and the AURA2 studies compare favorably with historical results. These data support the current recommendation of osimertinib for the treatment of patients with T790M-positive advanced NSCLC after progression with prior EGFR-TKI therapy. ${ }^{1}$
The response rates to osimertinib were similar across all patient subgroups regardless of the line of therapy or the specific prior EGFR-TKI treatment they had received. Of note, some patients had received many (up to 11) lines of cancer therapy before osimertinib. The response rate and PFS were numerically, although not statistically, higher for patients with co-occurring T790M and EGFR exon 19 deletion-activating mutations compared with those with co-occurring T790M and EGFR L858R mutations documented before the study start. Prior studies of early-generation EGFR-TKIs (gefitinib, erlotinib, and afatinib) demonstrated greater clinical benefit in patients who harbored an EGFR exon 19 deletion than in patients with an L858R mutation. ${ }^{20,21}$ We also observed a numerically higher response rate and longer PFS in Asian patients treated with osimertinib, similar to previous observations with early-generation EGFR-TKIs. ${ }^{3-6,8,22}$ The reasons behind these observations are not clear because prior pharmacokinetic analyses demonstrated that osimertinib exposure is similar in both Asian and non-Asian patients. ${ }^{23}$

Osimertinib was well tolerated and associated with a low incidence of discontinuations and dose reductions as a result of possibly causally related AEs. Osimertinib was associated with 

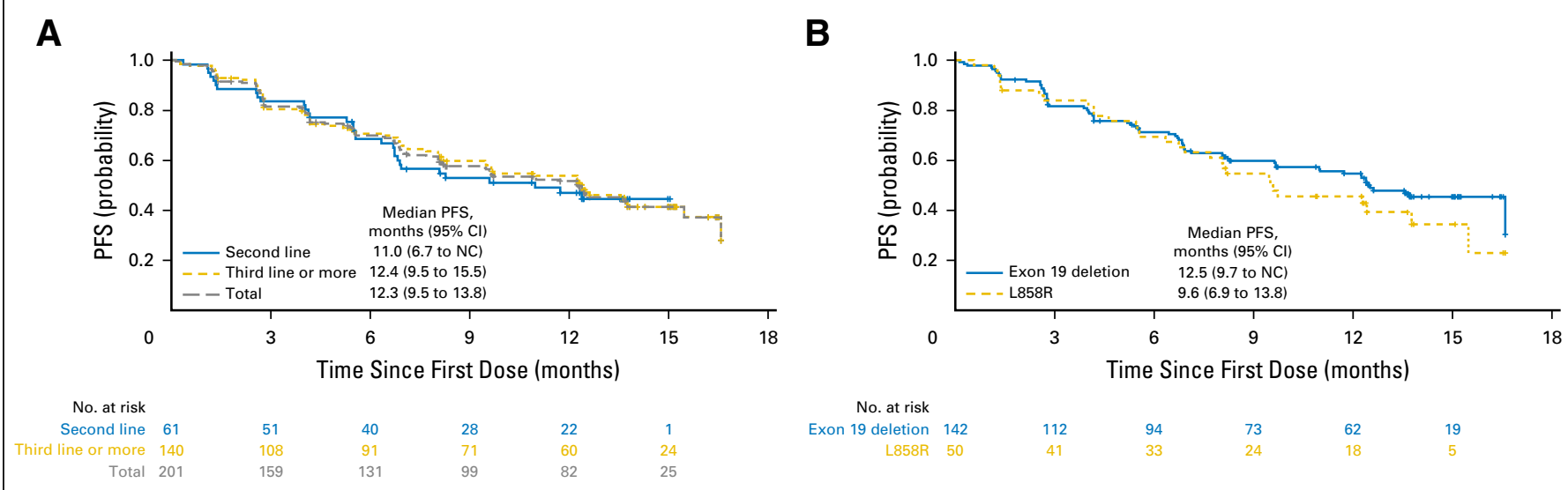

C

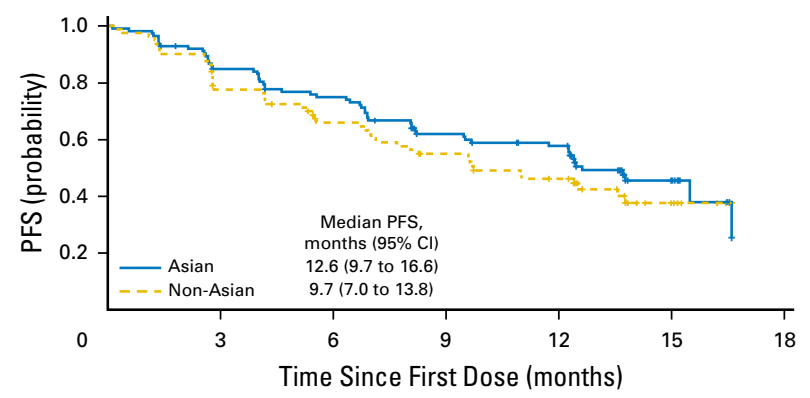

D
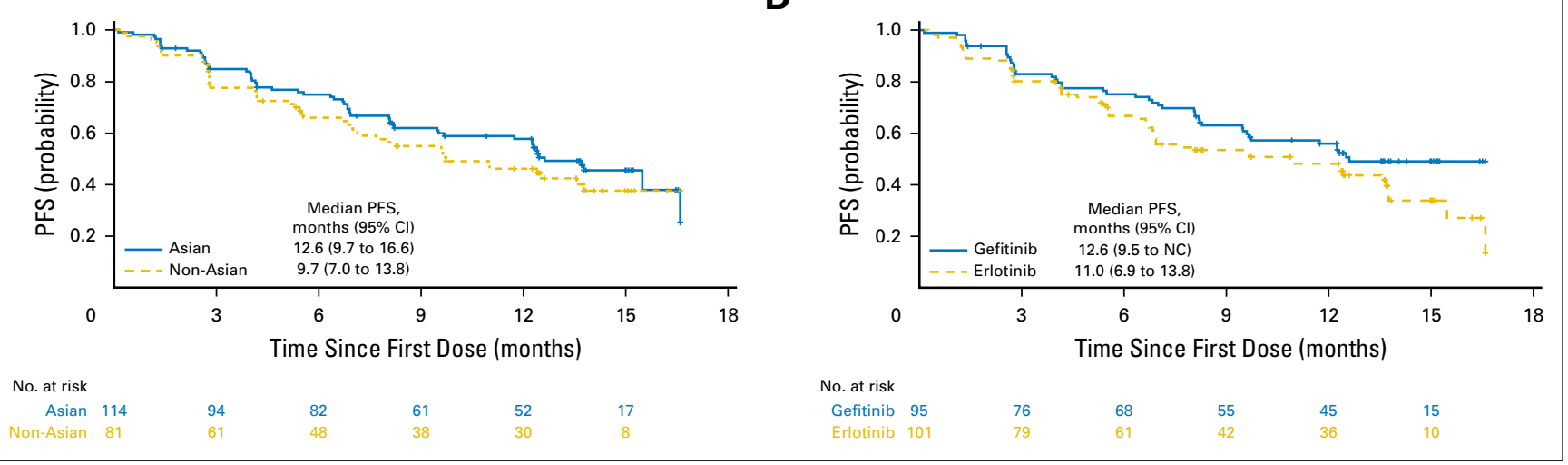

Fig 4. Progression-free survival (PFS) by blinded independent central review (full analysis set) by (A) line of therapy, by (B) co-occurring epidermal growth factor receptor

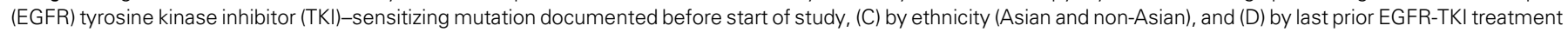
(gefitinib, erlotinib). Tick marks indicate censored observations. NC, not calculable.

symptomatic improvements, and PRO data in the AURA extension suggest that patients treated with osimertinib show benefits in symptoms, functioning, and health status/quality of life from baseline assessments.

ILD was observed in eight patients (4\%), and osimertinibassociated ILD was observed in both white and Asian patients. The mechanistic basis and risk factors that led to osimertinibassociated ILD remain unclear. Presently, patients in whom ILD develops should discontinue osimertinib therapy and receive appropriate supportive care.
For the AURA extension, central confirmation of T790M was carried out by using tumor tissue samples. In a recent study that analyzed plasma-derived circulating tumor DNA as a biomarker for osimertinib response, Oxnard et $\mathrm{al}^{24}$ concluded that patients positive for T790M in plasma have outcomes with osimertinib equivalent to patients with positive tissue-based assay results, which highlights the feasibility of plasma testing for selecting patients for osimertinib treatment. Recently, plasma-based T790M testing was approved by the US Food and Drug Administration. ${ }^{25}$

\begin{tabular}{|c|c|c|c|c|}
\hline \multirow[b]{2}{*}{ Possibly Causally Related Adverse Event* } & \multicolumn{4}{|c|}{ Grade, No. (\%) } \\
\hline & 1 & 2 & $\geq 3$ & Total \\
\hline Diarrhea & 74 (37) & $11(5)$ & $1(<1)$ & $86(43)$ \\
\hline Rash (grouped terms) & 67 (33) & $12(6)$ & $1(<1)$ & $80(40)$ \\
\hline Paronychia (grouped terms) & $46(23)$ & $17(8)$ & 0 & $63(31)$ \\
\hline Dry skin (grouped terms) & $56(28)$ & $6(3)$ & 0 & $62(31)$ \\
\hline Stomatitis & $21(10)$ & $6(3)$ & 0 & 27 (13) \\
\hline Pruritus & $21(10)$ & $6(3)$ & 0 & $27(13)$ \\
\hline Platelet count decreased & $23(11)$ & 0 & $2(1)$ & $25(12)$ \\
\hline Nausea & $18(9)$ & $3(1)$ & $1(<1)$ & $22(11)$ \\
\hline Decreased appetite & $16(8)$ & $3(1)$ & $1(<1)$ & $20(10)$ \\
\hline
\end{tabular}


Given that osimertinib is effective in treating patients with T790M-positive tumors and is a potent inhibitor of EGFRactivating mutations, the drug is under evaluation in patients with EGFR-TKI-naive advanced NSCLC. These studies will help to determine whether the development of T790M can be prevented and whether this approach is associated with a longer PFS than observed with gefitinib, erlotinib, or afatinib. Preliminary findings from a cohort of patients treated as part of the phase I trial demonstrated an encouraging response rate of $77 \%$ and a median PFS of 19.3 months. ${ }^{26} \mathrm{~A}$ randomized phase III trial to compare osimertinib with gefitinib and erlotinib in EGFR-TKI-naive advanced EGFRm NSCLC has completed enrollment (FLAURA [Osimertinib Versus Gefitinib or Erlotinib in Patients With Locally Advanced or Metastatic NSCLC]; NCT02296125). To date, the strategy of using a more potent kinase inhibitor capable of overcoming resistance mechanisms to existing kinase inhibitors as initial therapy, including alectinib in $A L K$-rearranged NSCLC or nilotinib or dasatinib in chronic myeloid leukemia, has resulted in improved patient outcomes. $^{27-29}$

In summary, the results from the AURA extension study show that osimertinib $80 \mathrm{mg}$ once daily provides high response rates, encouraging PFS, and a long DoR with a manageable safety profile in patients with T790M-positive pretreated advanced NSCLC.

\section{AUTHORS' DISCLOSURES OF POTENTIAL CONFLICTS} OF INTEREST

Disclosures provided by the authors are available with this article at jco.org.

\section{AUTHOR CONTRIBUTIONS}

Conception and design: James Chih-Hsin Yang, Suresh S. Ramalingam, Silvia Novello, Mireille Cantarini, Serban Ghiorghiu

Provision of study materials or patients: James Chih-Hsin Yang, DongWan Kim, Lecia V. Sequist, Sang-We Kim, Enriqueta Felip, Kiyotaka Yoh, Silvia Novello, Kathryn Gold, Tomonori Hirashima, Serban Ghiorghiu, Pasi A. Jänne Collection and assembly of data: James Chih-Hsin Yang, Myung-Ju Ahn, Dong-Wan Kim, Suresh S. Ramalingam, Lecia V. Sequist,Wu-Chou Su, Sang-We Kim, Joo-Hang Kim, Fiona Blackhall, Silvia Novello, Kathryn Gold, Tomonori Hirashima, Chia-Chi Lin, Mireille Cantarini, Serban Ghiorghiu, Pasi A. Jänne

Data analysis and interpretation: James Chih-Hsin Yang, Myung-Ju Ahn, Dong-Wan Kim, Suresh S. Ramalingam, Lecia V. Sequist, Joo-Hang Kim, David Planchard, Enriqueta Felip, Daniel Haggstrom, Kiyotaka Yoh, Silvia Novello, Kathryn Gold, Chia-Chi Lin, Helen Mann, Mireille Cantarini, Serban Ghiorghiu, Pasi A. Jänne

Manuscript writing: All authors

Final approval of manuscript: All authors

Accountable for all aspects of the work: All authors

\section{REFERENCES}

1. National Comprehensive Cancer Network: NCCN clinical practice guidelines in oncology: NSCLC (version 4), 2016. http://www.nccn.org/professionals/ physician_gls/pdf/nscl.pdf

2. Reck M, Popat $S$, Reinmuth $N$, et al: Metastatic non-small-cell lung cancer (NSCLC): ESMO clinical practice guidelines for diagnosis, treatment and follow-up. Ann Oncol 25:iii27-iii39, 2014 (suppl 3)

3. Mok TS, Wu YL, Thongprasert $S$, et al: Gefitinib or carboplatin-paclitaxel in pulmonary adenocarcinoma. N Engl J Med 361:947-957, 2009

4. Maemondo $M$, Inoue $A$, Kobayashi $K$, et al: Gefitinib or chemotherapy for non-small-cell lung cancer with mutated EGFR. N Engl J Med 362: 2380-2388, 2010

5. Rosell R, Carcereny E, Gervais R, et al: Erlotinib versus standard chemotherapy as first-line treatment for European patients with advanced EGFR mutation-positive non-small-cell lung cancer (EURTAC): A multicentre, open-label, randomised phase 3 trial. Lancet Oncol 13:239-246, 2012

6. Sequist LV, Yang JC, Yamamoto $N$, et al: Phase III study of afatinib or cisplatin plus pemetrexed in patients with metastatic lung adenocarcinoma with EGFR mutations. J Clin Oncol 31: 3327-3334, 2013

7. Wu Y-L, Zhou C, Hu C-P, et al: Afatinib versus cisplatin plus gemcitabine for first-line treatment of Asian patients with advanced non-small-cell lung cancer harbouring EGFR mutations (LUX-Lung 6): An open-label, randomised phase 3 trial. Lancet Oncol 15:213-222, 2014

8. Zhou $\mathrm{C}, \mathrm{Wu}$ YL, Chen G, et al: Erlotinib versus chemotherapy as first-line treatment for patients with advanced EGFR mutation-positive non-small-cell lung cancer (OPTIMAL, CTONG-0802): A multicentre, open-label, randomised, phase 3 study. Lancet Oncol 12:735-742, 2011

9. Arcila ME, Oxnard GR, Nafa K, et al: Rebiopsy of lung cancer patients with acquired resistance to EGFR inhibitors and enhanced detection of the T790M mutation using a locked nucleic acid-based assay. Clin Cancer Res 17:1169-1180, 2011

10. Oxnard GR, Arcila ME, Sima CS, et al: Acquired resistance to EGFR tyrosine kinase in hibitors in EGFR-mutant lung cancer: Distinct natural history of patients with tumors harboring the T790M mutation. Clin Cancer Res 17: 1616-1622, 2011

11. Sun JM, Ahn MJ, Choi $Y L$, et al: Clinical implications of T790M mutation in patients with acquired resistance to EGFR tyrosine kinase inhibitors. Lung Cancer 82:294-298, 2013

12. Yu HA, Arcila ME, Rekhtman N, et al: Analysis of tumor specimens at the time of acquired resistance to EGFR-TKI therapy in 155 patients with EGFR-mutant lung cancers. Clin Cancer Res 19: 2240-2247, 2013

13. Cross DA, Ashton SE, Ghiorghiu $S$, et al: AZD9291, an irreversible EGFR TKI, overcomes T790M-mediated resistance to EGFR inhibitors in lung cancer. Cancer Discov 4:1046-1061, 2014

14. Jänne PA, Yang JC, Kim DW, et al: AZD9291 in EGFR inhibitor-resistant non-small-cell lung cancer. N Engl J Med 372:1689-1699, 2015

15. Goss G, Tsai CM, Shepherd FA, et al: Osimertinib for pretreated EGFR Thr790Met-positive advanced non-small-cell lung cancer (AURA2): A multicentre, open-label, single-arm, phase 2 study. Lancet Oncol 17:1643-1652, 2016

16. Brown PD, Jaeckle $K$, Ballman KV, et al: Effect of radiosurgery alone vs radiosurgery with whole brain radiation therapy on cognitive function in patients with 1 to 3 brain metastases: A randomized clinical trial. JAMA 316:401-409, 2016
17. Soria JC, Wu YL, Nakagawa K, et al: Gefitinib plus chemotherapy versus placebo plus chemotherapy in EGFR-mutation-positive non-small-cell lung cancer after progression on first-line gefitinib (IMPRESS): A phase 3 randomised trial. Lancet Oncol 16:990-998, 2015

18. Hu S, Mohan Kumar D, Sax C, et al: Pseudotyping of lentiviral vector with novel vesiculovirus envelope glycoproteins derived from Chandipura and Piry viruses. Virology 488:162-168, 2016

19. Janjigian $Y Y$, Smit EF, Groen $H J$, et al: Dua inhibition of EGFR with afatinib and cetuximab in kinase inhibitor-resistant EGFR-mutant lung cancer with and without T790M mutations. Cancer Discov 4: 1036-1045, 2014

20. Lee $C K, W u Y L$, Ding $P N$, et al: Impact of specific epidermal growth factor receptor (EGFR) mutations and clinical characteristics on outcomes after treatment with EGFR tyrosine kinase inhibitors versus chemotherapy in EGFR-mutant lung cancer: A meta-analysis. J Clin Oncol 33:1958-1965, 2015

21. Jackman DM, Yeap BY, Sequist LV, et al: Exon 19 deletion mutations of epidermal growth factor receptor are associated with prolonged survival in non-small cell lung cancer patients treated with gefitinib or erlotinib. Clin Cancer Res 12:3908-3914, 2006

22. Douillard JY, Ostoros G, Cobo M, et al: Firstline gefitinib in Caucasian EGFR mutation-positive NSCLC patients: A phase-IV, open-label, single-arm study. Br J Cancer 110:55-62, 2014

23. Planchard D, Brown KH, Kim DW, et al: Osimertinib western and Asian clinical pharmacokinetics in patients and healthy volunteers: Implications for formulation, dose, and dosing frequency in pivotal clinical studies. Cancer Chemother Pharmacol 77 767-776, 2016

24. Oxnard GR, Thress KS, Alden RS, et al: Association between plasma genotyping and outcomes 
of treatment with osimertinib (AZD9291) in advanced non-small-cell lung cancer. J Clin Oncol 34: 3375-3382, 2016

25. AstraZeneca: US FDA approves TAGRISSO ${ }^{\circledR}$ (osimertinib) blood-based T790M companion diagnostic test, September 29, 2016. https://www. astrazeneca-us.com/media/press-releases/2016/usfda-approves-tagrisso-osimertinib-blood-based-t790mcompanion-diagnostic-test-09292016.htm
26. Ramalingam $\mathrm{S}$, Yang $\mathrm{JCH}$, Lee $\mathrm{CK}$, et al: LBA1_PR: Osimertinib as first-line treatment for EGFR mutation-positive advanced NSCLC: Updated efficacy and safety results from two phase I expansion cohorts. J Thorac Oncol 11:S152, 2016 (suppl 4)

27. Kantarjian H, Giles F, Wunderle L, et al: Nilotinib in imatinib-resistant $\mathrm{CML}$ and Philadelphia chromosome-positive ALL. N Engl J Med 354: 2542-2551, 2006
28. Shaw AT, Gandhi L, Gadgeel S, et al: Alectinib in ALK-positive, crizotinib-resistant, nonsmall-cell lung cancer: a single-group, multicentre, phase 2 trial. Lancet Oncol 17:234-242, 2016

29. Talpaz M, Shah NP, Kantarjian H, et al: Dasatinib in imatinib-resistant Philadelphia chromosomepositive leukemias. N Engl J Med 354:2531-2541, 2006

\section{Affiliations}

James Chih-Hsin Yang and Chia-Chi Lin, National Taiwan University Hospital, Taipei; Wu-Chou Su, National Cheng Kung University Hospital, Tainan, Taiwan, Republic of China; Myung-Ju Ahn, Sungkyunkwan University; Dong-Wan Kim, Seoul National University Hospital; Sang-We Kim, Asan Medical Center, Seoul; Joo-Hang Kim, CHA University, Gyeonggi-do, Republic of Korea; Suresh S. Ramalingam, Emory University School of Medicine, Atlanta, GA; Lecia V. Sequist, Massachusetts General Hospital; Pasi A. Jänne, Dana-Farber Cancer Institute, Boston, MA; David Planchard, Institut Gustave Roussy, Villejuif, France; Enriqueta Felip, Vall d'Hebron University Hospital, Barcelona, Spain; Fiona Blackhall, The Christie Hospital; University of Manchester, Manchester; Helen Mann and Serban Ghiorghiu, AstraZeneca, Cambridge; Mireille Cantarini, AstraZeneca, Macclesfield, United Kingdom; Daniel Haggstrom, Carolinas Healthcare System, Charlotte, NC; Kiyotaka Yoh, National Cancer Center Hospital East, Kashiwa, Chiba; Tomonori Hirashima, Osaka Prefectural Medical Center for Respiratory and Allergic Diseases, Osaka, Japan; Silvia Novello, University of Turin, Turin, Italy; and Kathryn Gold, University of California San Diego Moores Cancer Center, San Diego, CA.

Supported by AstraZeneca.

\section{Support}




\section{AUTHORS' DISCLOSURES OF POTENTIAL CONFLICTS OF INTEREST}

Osimertinib in Pretreated T790M-Positive Advanced Non-Small-Cell Lung Cancer: AURA Study Phase II Extension Component

The following represents disclosure information provided by authors of this manuscript. All relationships are considered compensated. Relationships are self-held unless noted. I = Immediate Family Member, Inst = My Institution. Relationships may not relate to the subject matter of this manuscript. For more information about ASCO's conflict of interest policy, please refer to www.asco.org/rwc or ascopubs.org/jco/site/ifc.

\section{James Chih-Hsin Yang}

Honoraria: Boehringer Ingelheim, Roche, Chugai Pharmaceutical, MSD, AstraZeneca, Novartis

Consulting or Advisory Role: Boehringer Ingelheim, Novartis, AstraZeneca, Roche, Genentech, Clovis Oncology, Eli Lilly, MSD, Merck Serono, Celgene, Astellas Pharma, Bayer AG, Pfizer, Ono Pharmaceutical, Bristol-Myers Squibb, Boehringer Ingelheim (Inst), AstraZeneca (Inst), Yuhan, Daiichi Sankyo

\section{Myung-Ju Ahn}

No relationship to disclose

\section{Dong-Wan Kim}

No relationship to disclose

Suresh S. Ramalingam

Honoraria: AstraZeneca, Boehringer Ingelheim, Genentech Consulting or Advisory Role: AstraZeneca, Boehringer Ingelheim, Genentech

\section{Lecia V. Sequist}

Consulting or Advisory Role: Clovis Oncology, Novartis, Merrimack Pharmaceuticals, AstraZeneca, ARIAD Pharmaceuticals, Genentech, Roche, Bristol-Myers Squibb

Research Funding: Boehringer Ingelheim (Inst), Clovis Oncology (Inst), Genentech (Inst), Merrimack Pharmaceuticals (Inst), Novartis (Inst), AstraZeneca (Inst), Johnson \& Johnson (Inst), Eli Lilly (Inst), Merck (Inst), Taiho Pharmaceutical (Inst)

\section{Wu-Chou Su}

Travel, Accommodations, Expenses: Roche (I), MSD (I)

\section{Sang-We Kim}

No relationship to disclose

\section{Joo-Hang Kim}

Research Funding: Roche (Inst), AstraZeneca (Inst), Bristol-Myers Squibb (Inst), Eli Lilly (Inst), MSD (Inst)

David Planchard

Consulting or Advisory Role: AstraZeneca, Roche, MSD, Pfizer, Boehringer Ingelheim, Eli Lilly, Novartis, Bristol-Myers Squibb Research Funding: Novartis (Inst)

\section{Enriqueta Felip}

Consulting or Advisory Role: Eli Lilly, Pfizer, Roche, Boehringer Ingelheim, MSD

Speakers' Bureau: AstraZeneca, Bristol-Myers Squibb, Novartis

Fiona Blackhall

Honoraria: Medivation, AstraZeneca

Consulting or Advisory Role: Medivation, AstraZeneca

Research Funding: Pfizer (Inst), AstraZeneca (Inst), Boehringer

Ingelheim (Inst), Novartis (Inst)

\section{Daniel Haggstrom}

No relationship to disclose

\section{Kiyotaka Yoh}

Honoraria: Chugai Pharmaceutical, AstraZeneca, Eli Lilly Japan,

Boehringer Ingelheim, Pfizer, Taiho Pharmaceutical, Bristol-Myers Squibb, Ono Pharmaceutical

Consulting or Advisory Role: Boehringer Ingelheim, AstraZeneca, Eli Lilly Japan

Research Funding: Eli Lilly Japan (Inst), Bayer AG (Inst), Novartis (Inst), AstraZeneca (Inst), Takeda Pharmaceuticals (Inst), Pfizer (Inst)

\section{Silvia Novello}

Speakers' Bureau: AstraZeneca, MSD, Bristol-Myers Squibb, Roche, Pfizer, Eli Lilly

\section{Kathryn Gold}

Honoraria: Roche, Genentech

Consulting or Advisory Role: Pfizer, ARIAD Pharmaceuticals Research Funding: AstraZeneca, ARIAD Pharmaceuticals, Bristol-Myers Squibb, Pharmacyclics, Roche, Genentech, Astellas Pharma

Travel, Accommodations, Expenses: AstraZeneca

\section{Tomonori Hirashima}

Research Funding: MSD, AstraZeneca, Eisai, Daiichi Sankyo, Merck Serono, Eli Lilly Japan, Taiho Pharmaceutical, Kyowa Hakko Kirin, Takeda, Boehringer Ingelheim

\section{Chia-Chi Lin}

No relationship to disclose

Helen Mann

Employment: AstraZeneca

Stock or Other Ownership: AstraZeneca (I)

\section{Mireille Cantarini}

Employment: AstraZeneca

Stock or Other Ownership: AstraZeneca

\section{Serban Ghiorghiu}

Employment: AstraZeneca, AstraZeneca (I)

Stock or Other Ownership: AstraZeneca, AstraZeneca (I)

Pasi A. Jänne

Stock or Other Ownership: Gatekeeper Pharmaceuticals

Consulting or Advisory Role: AstraZeneca, Boehringer Ingelheim, Pfizer, Chugai Pharmaceutical, ARIAD Pharmaceuticals, Merrimack Pharmaceuticals, Roche, Genentech, Loxo Oncology, Ignyta Research Funding: Astellas Pharma, AstraZeneca

Patents, Royalties, Other Intellectual Property: LabCorp patent for postmarketing royalties from institution-owned intellectual property on EGFR mutations licensed to LabCorp 


\section{Acknowledgment}

J.C.Y. drafted the manuscript, and Natasha Cary from iMed Comms, an Ashfield Company, provided editorial assistance funded by AstraZeneca. We thank Rachael Lawrance for statistical input. We thank all 40 sites that helped with screening, the investigators, and the patients and their families who participated in this study.

\section{Appendix}

\section{Study Design and Participants}

Eligibility criteria included the prospective confirmation of EGFR T790M mutation status by a central laboratory that used the cobas EGFR Mutation Test (Roche Molecular Systems, Pleasanton, CA) from a tissue biopsy specimen taken after confirmed disease progression while receiving the most recent treatment regimen (irrespective of whether epidermal growth factor receptor [EGFR] tyrosine kinase inhibitor [TKI] or chemotherapy), a WHO performance status of 0 to 1 , acceptable organ function, and at least one measurable lesion not previously irradiated or chosen for biopsy during study screening. Patients with spinal cord compression or brain metastases could be enrolled if the disease was asymptomatic, stable, and not requiring corticosteroids for at least 4 weeks before first dose of study treatment.

Patients were excluded from the study if they had received treatment with an EGFR-TKI within 8 days or 5 half-lives of the first dose of study treatment; chemotherapy, investigational agents, or other anticancer drugs from a previous regimen within 14 days of the first dose of study treatment and medications or supplements known to inhibit cytochrome P450 isoenzyme 2C8 and inhibitors or inducers of cytochrome P450 isoenzyme 3A4 within 7 days of the first dose of study treatment. Patients with unresolved toxicity from prior treatment of more than National Cancer Institute Common Terminology Criteria for Adverse Events (version 4 ) grade 1 were excluded. Patients with a medical history of interstitial lung disease (ILD), drug-induced ILD, radiation pneumonitis that required corticosteroid treatment, or any evidence of clinically active ILD also were excluded.

\section{cobas EGFR Mutation Test}

The limit of detection for EGFR T790M detection with the cobas EGFR Mutation Test version 1 is $2.04 \%$ (O'Donnell et al: BMC Cancer 13:210, 2013). Compared with next-generation sequencing (two-tube amplicon assay run on MiSeq, read depth $\times 5,000$; Roche Molecular Systems), positive and negative percent agreements for EGFR T790M detection were 91\% and 97\%, respectively (Appendix Table A5, online only).

\section{Procedures}

If a patient experienced a Common Terminology Criteria for Adverse Events (version 4) grade 3 or an unacceptable adverse event, osimertinib dosing was interrupted for up to 21 days. If the toxicity resolved to grade $\leq 2$ within 21 days and the patient showed clinical benefit, treatment could be restarted at the same or lower dose of $40 \mathrm{mg} /$ day. Patients with persistent QTc prolongation for $>21$ days or ILD were permanently withdrawn from study treatment but followed up postdiscontinuation for disease progression.

Patient-reported outcomes were collected by using the European Organization for Research and Treatment of Cancer questionnaires QLQ-LC13 (disease-specific symptoms) and QLQ-C30 (general cancer symptoms, functioning, and global health status/quality of life) completed at baseline and then every 6 weeks.

\section{Definitions of Progression-Free Survival and Duration of Response}

Progression-free survival was defined as the time from date of first dose until the date of objective disease progression or death, regardless of whether the patient withdrew from study treatment or received another anticancer therapy before progression. Duration of response was defined as the time from date of first documented response (subsequently confirmed) until date of documented progression or death in the absence of disease progression. 
Yang et al

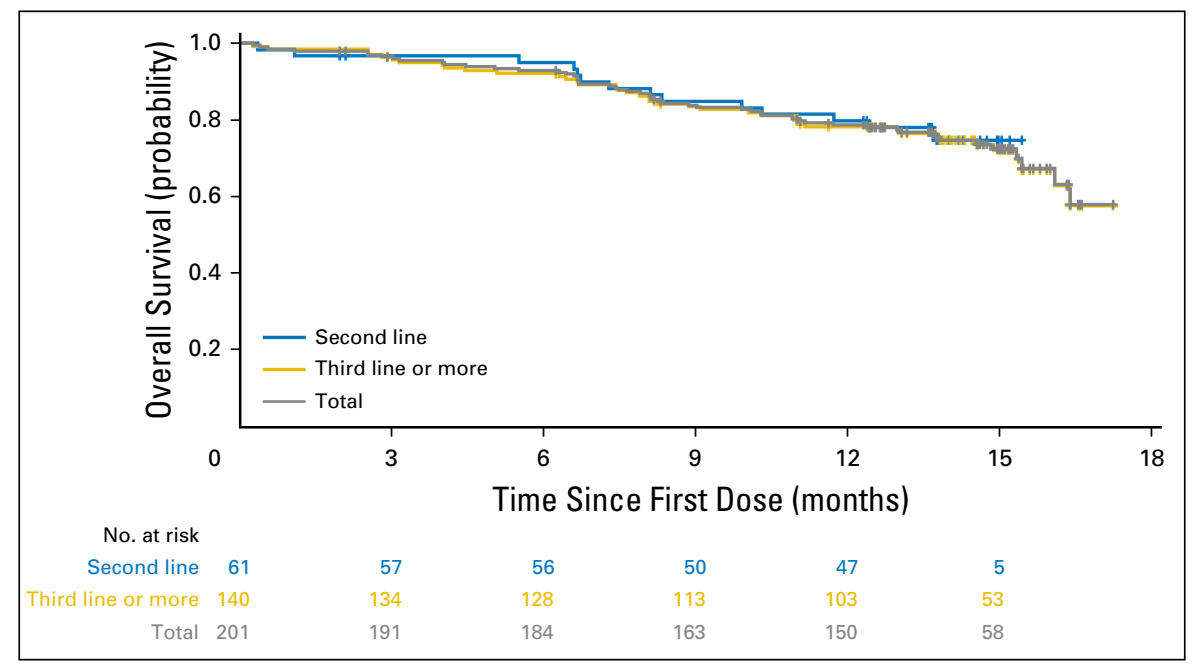

Fig A1. Overall survival by line of therapy. 


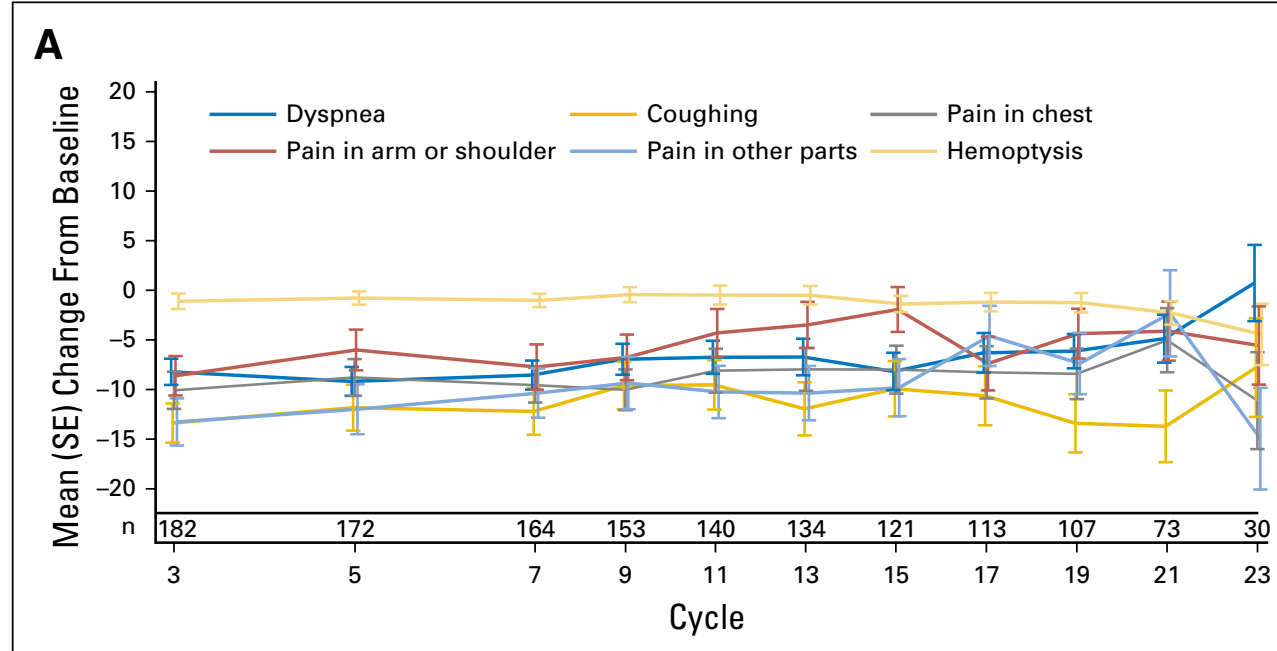

B

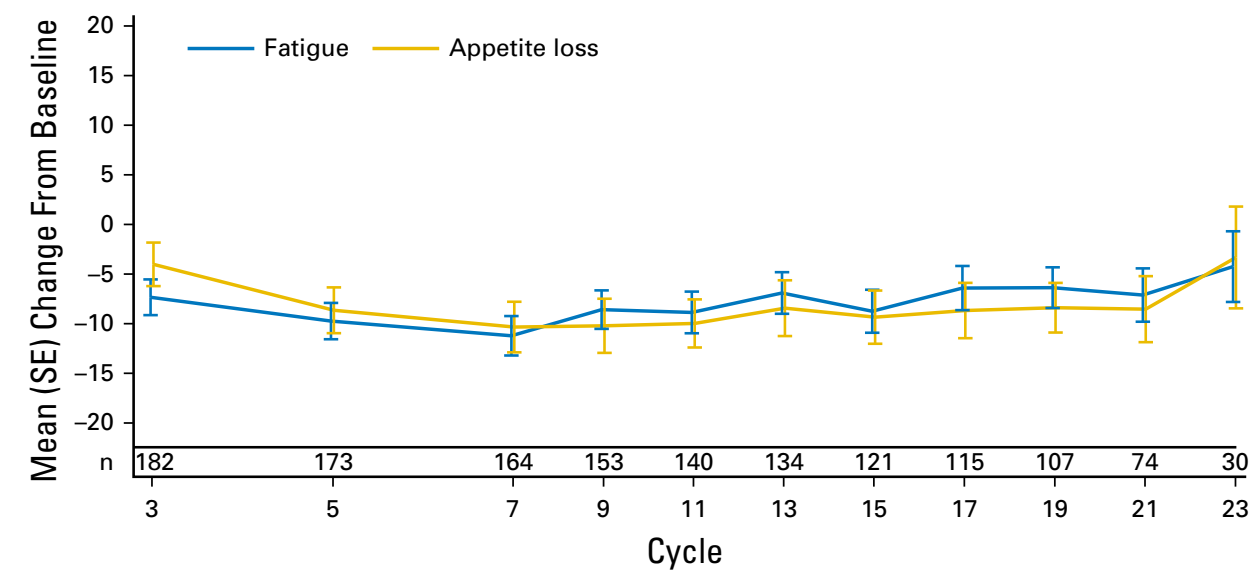

C

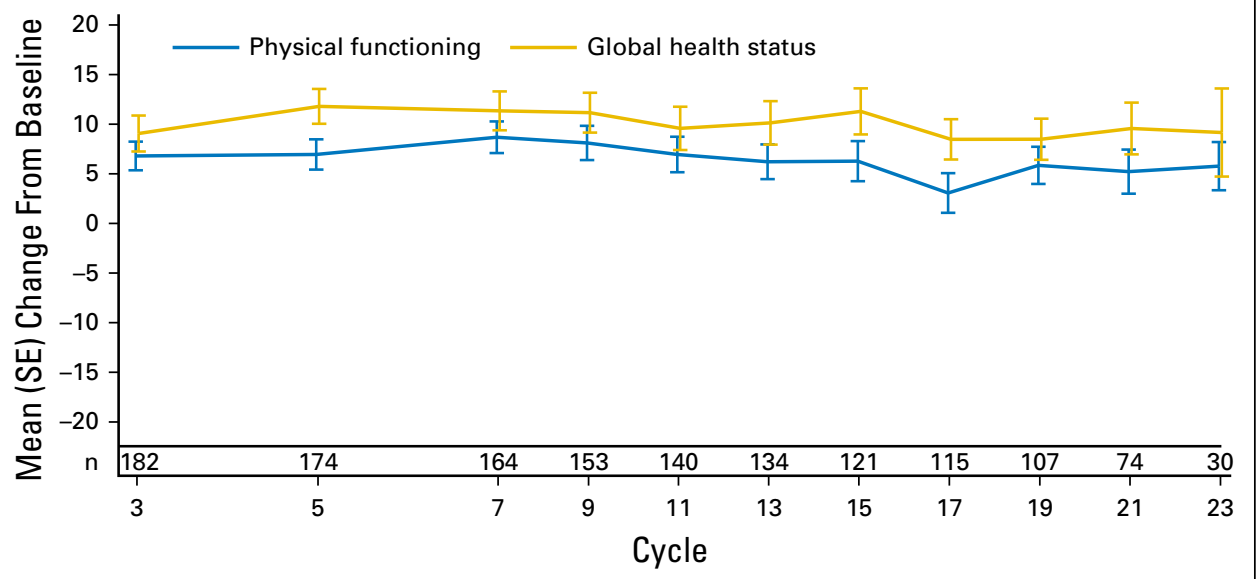

Fig A2. Patients showed consistent and sustained improvements in key lung cancer symptoms, including (A) dyspnea, cough, pain, and hemoptysis; (B) fatigue and appetite loss; and (C) physical functioning and global health status. 


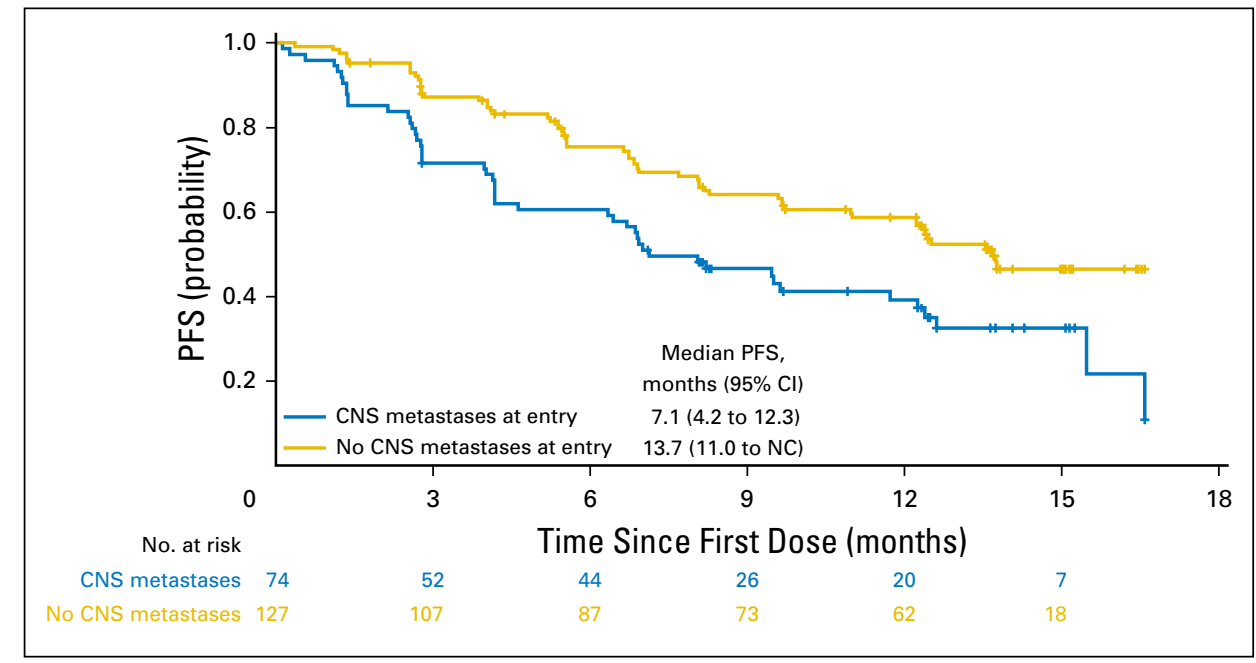

Fig A3. Progression-free survival (PFS) by CNS metastases at study entry. Tick marks indicate censored observations.

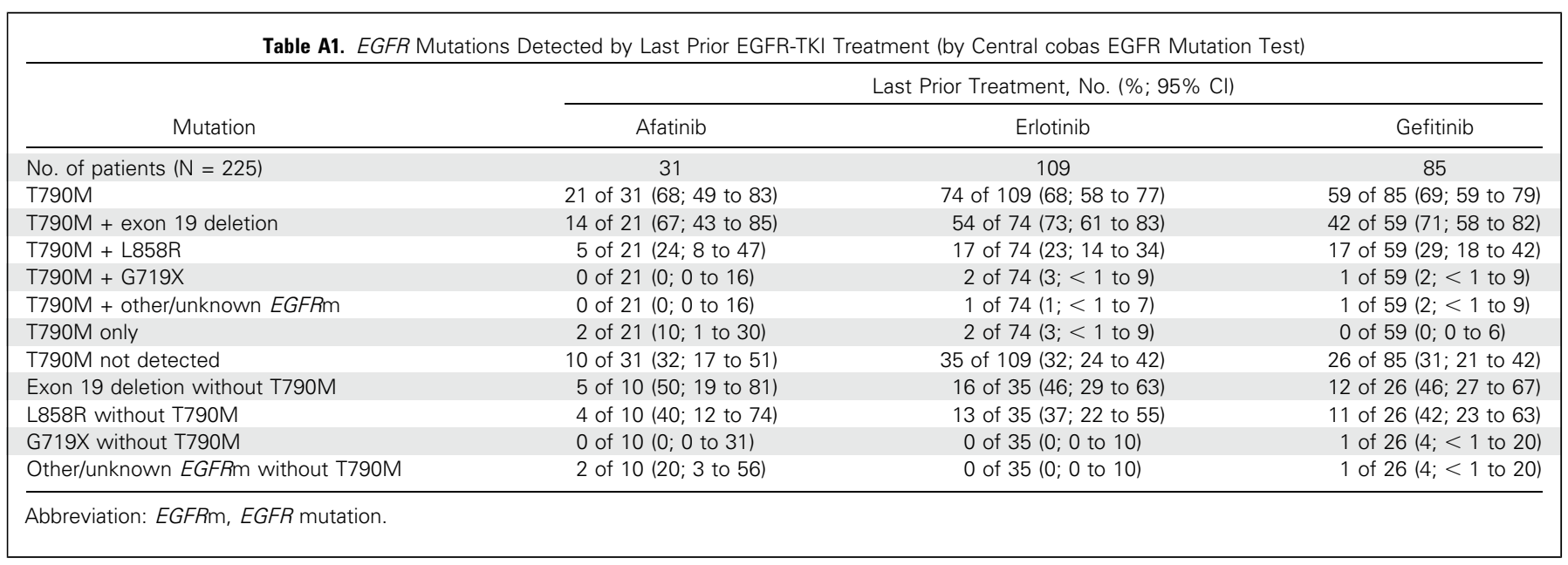




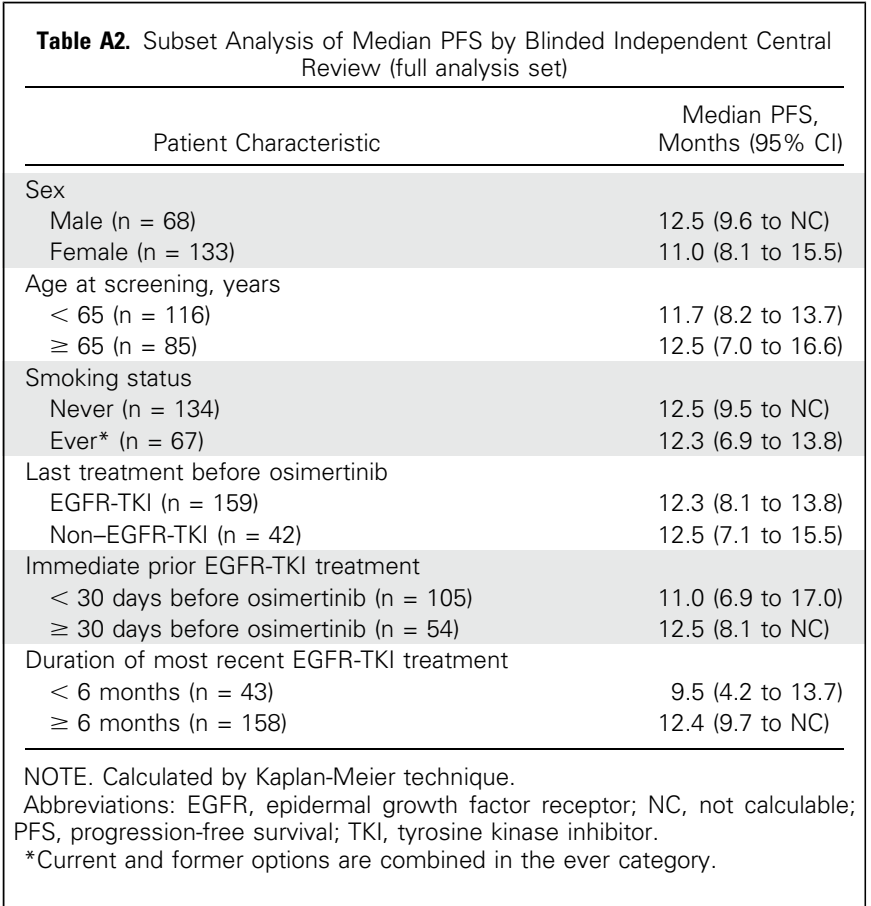

\begin{tabular}{|c|c|}
\hline AE Category* & No. $(\%) \dagger$ \\
\hline No. of patients & 201 \\
\hline Any $A E$ & $199(99)$ \\
\hline Any possibly causally related $\mathrm{AE} \neq$ & $185(92)$ \\
\hline Any grade $\geq 3 \mathrm{AE}$ & 77 (38) \\
\hline Any grade $\geq 3$ possibly causally related $A E \ddagger$ & $30(15)$ \\
\hline Any AE with outcome of death & $7(3)$ \\
\hline Any possibly causally related AE with outcome of death $\neq$ & $3(1)$ \\
\hline Any SAE (including events with outcome of death) & $55(27)$ \\
\hline $\begin{array}{l}\text { Any possibly causally related SAE (including events with } \\
\text { outcome of death) } \neq\end{array}$ & $12(6)$ \\
\hline Any $A E$ that led to interruption of osimertinib & $43(21)$ \\
\hline Any $A E$ that led to reduction of osimertinib & $10(5)$ \\
\hline Any $A E$ that led to discontinuation of osimertinib & $15(7)$ \\
\hline $\begin{array}{l}\text { Any possibly causally related } A E \text { that led to discontinuation of } \\
\text { osimertinib } \neq\end{array}$ & $9(4)$ \\
\hline \multicolumn{2}{|c|}{$\begin{array}{l}\text { Abbreviations: AE, adverse event; SAE, serious adverse event. } \\
\text { *Includes adverse events with an onset date on or after the date of first dose } \\
\text { and up to and including } 28 \text { days following the date of the last dose of study } \\
\text { medication. } \\
\text { †Patients with multiple events in the same category are counted only once in } \\
\text { that category. Patients with events in more than one category are counted once } \\
\text { in each of those categories. } \\
\neq \text { As assessed by the investigator. }\end{array}$} \\
\hline
\end{tabular}




\begin{tabular}{|c|c|c|c|c|}
\hline \multirow[b]{2}{*}{$A E^{*}$} & \multicolumn{4}{|c|}{ Grade, No. (\%) } \\
\hline & 1 & 2 & $\geq 3$ & Total $(N=201)$ \\
\hline Diarrheat & 78 (39) & $16(8)$ & $2(1)$ & $97(48) \S$ \\
\hline Rash (grouped terms) & $74(37)$ & $14(7)$ & $1(<1)$ & $89(44)$ \\
\hline Dry skin (grouped terms) & $60(30)$ & $7(3)$ & 0 & 67 (33) \\
\hline Paronychia (grouped terms) & $47(23)$ & $18(9)$ & 0 & $65(32)$ \\
\hline Decreased appetite & $32(16)$ & $8(4)$ & $2(1)$ & $42(21)$ \\
\hline Cough & $35(17)$ & $6(3)$ & 0 & $41(20)$ \\
\hline Nausea & $30(15)$ & $8(4)$ & $3(1)$ & $41(20)$ \\
\hline Constipation & $31(15)$ & $7(3)$ & $1(<1)$ & 39 (19) \\
\hline Back pain & $26(13)$ & $8(4)$ & $1(<1)$ & $35(17)$ \\
\hline Stomatitis & $26(13)$ & $6(3)$ & 0 & $32(16)$ \\
\hline Platelet count decreased & $26(13)$ & $3(1)$ & $2(1)$ & $31(15)$ \\
\hline Pruritus & $25(12)$ & $6(3)$ & 0 & $31(15)$ \\
\hline Anemia & $13(6)$ & $10(5)$ & 7 (3) & $30(15)$ \\
\hline Fatigue & $14(7)$ & $12(6)$ & $2(1)$ & $28(14)$ \\
\hline Vomiting & $20(10)$ & $5(2)$ & $3(1)$ & $28(14)$ \\
\hline Headache & $25(12)$ & $1(<1)$ & $1(<1)$ & $27(13)$ \\
\hline Asthenia & $12(6)$ & $7(3)$ & $5(2)$ & $24(12)$ \\
\hline Dyspnea & $12(6)$ & $7(3)$ & $5(2)$ & $24(12)$ \\
\hline Insomnia & $20(10)$ & $4(2)$ & 0 & $24(12)$ \\
\hline Upper respiratory infection & $16(8)$ & $8(4)$ & 0 & $24(12)$ \\
\hline
\end{tabular}

\begin{tabular}{|c|c|c|c|c|c|}
\hline Test Result & Positive & Negative & Invalid & No Sample & Total \\
\hline Positive & 190 & 3 & 0 & 14 & 207 \\
\hline Negative & 18 & 82 & 0 & 17 & 117 \\
\hline Invalid & 0 & 0 & 0 & 3 & 3 \\
\hline \multicolumn{6}{|c|}{ Without invalid results, \% $(95 \% \mathrm{Cl})$} \\
\hline Positive percent agreement & & & 91 (87 to 95$)$ & & \\
\hline Negative percent agreement & & & 97 (90 to 99 ) & & \\
\hline Overall percent agreement & & & 93 (89 to 96 ) & & \\
\hline
\end{tabular}

*Two-tube amplicon assay run on MiSeq, read depth ×5,000 (Roche Molecular Systems, Pleasanton, CA). 